\title{
Deterministic Teleportation of Electrons in a Quantum Dot Nanostructure
}

\author{
R. L. de Visser and M. Blaauboer \\ Kavli Institute of Nanoscience, Delft University of Technology, Lorentzweg 1, 2628 CJ Delft, The Netherlands
}

(Received 10 January 2006; published 19 June 2006)

\begin{abstract}
We present a proposal for deterministic quantum teleportation of electrons in a semiconductor nanostructure consisting of a single and a double quantum dot. The central issue addressed in this Letter is how to design and implement the most efficient-in terms of the required number of single and two-qubit operations - deterministic teleportation protocol for this system. Using a group-theoretical analysis, we show that deterministic teleportation requires a minimum of three single-qubit rotations and two entangling $(\sqrt{\mathrm{SWAP}})$ operations. These can be implemented for spin qubits in quantum dots using electron-spin resonance (for single-spin rotations) and exchange interaction (for $\sqrt{\text { SWAP }}$ operations).
\end{abstract}

DOI: 10.1103/PhysRevLett.96.246801

Quantum teleportation is the process whereby a quantum state is transported from one place to another without moving through the intervening space. This process, proposed by Bennett et al. [1], involves a source particle (whose quantum state is to be transported) plus two entangled particles at a distant location, one of which is the target particle (to which the quantum state will be transferred). The teleportation procedure is then as follows: The two entangled particles are separated, and one of them is sent to "Alice," who also holds the source particle, while the other is sent to "Bob." Alice performs a joint measurement on her two particles in the Bell basis and communicates the result to Bob. Depending on this information, Bob applies one out of four unitary operations to his particle, which thereby acquires the original quantum state of the source particle. If Alice can distinguish all four possible measurement outcomes, the teleportation process can, in principle, be completed with a $100 \%$ success rate and is called deterministic. If Alice, on the other hand, is only able to perform a partial measurement on her two particles, the success probability is less than 1 and teleportation is probabilistic.

Probabilistic teleportation has been experimentally demonstrated for photons [2] and from one atom to another within the same molecule [3]. Deterministic quantum teleportation has been achieved for optical fields [4] and atomic ions [5]. Electrons have not been teleported yet. In recent years, several theoretical proposals have appeared for quantum teleportation of electrons, using excitons in coupled quantum dots [6,7], electron spins in a circuit consisting of normal and superconducting quantum dots [8], electron-hole pairs in a two-dimensional electron gas [9], charge qubits in a quantum dot array [10], and entangled electron-photon-electron states [11]. Out of these proposals, Refs. $[6,11]$ are, in principle, deterministic.

Here we propose the first all-electronic deterministic teleportation scheme for electron spins in a quantum dot nanostructure [12]. This choice of system is motivated by: (i) the high level of control over the number of electrons confined in quantum dots [13], (ii) recent advances in coherent manipulation of single and entangled pairs of spins
PACS numbers: 73.63.-b, 03.65.Ud, 03.67.Hk, 85.35.Be

in these structures [14], and (iii) the relative robustness of the electron spin against decoherence [15]. Compared to photons and ions, however, the coherence time $\left(\mathrm{T}_{2}\right)$ of electron spins in quantum dots - although it has not been measured yet for a single spin - is expected to be orders of magnitude shorter and forms the primary limiting factor for coherent quantum communication processes such as teleportation. The central question we ask in this Letter, therefore, is how to design the most efficient, i.e., the least time-consuming, scheme for deterministic teleportation of electron spins. To this end, we first examine the teleportation protocol of Bennett et al. [1] and note that Alice has some freedom in the choice of her measurement basis: It does not need to be the Bell basis, any maximally entangled basis will do. We then exploit this freedom to derive the general form of evolution operators which transform a maximally entangled basis into the standard basis $\{|\uparrow \uparrow\rangle,|\uparrow \downarrow\rangle,|\downarrow \uparrow\rangle,|\downarrow \downarrow\rangle\}$, which is the natural measurement basis in quantum dots. Our key result is the demonstration that such an evolution operator (and, hence, Alice's full measurement) cannot be implemented using less than one single-spin rotation plus two $\sqrt{\mathrm{SWAP}}$ operations.

The Letter is organized as follows. First, we briefly review the original teleportation protocol [1] and propose a straightforward implementation of this protocol for probabilistic teleportation of electron spins in a quantum dot nanostructure. We then describe how this probabilistic scheme should be modified in order to be able to achieve deterministic teleportation and present our proposal. The Letter ends with a discussion of the various time scales involved in the teleportation process and a comparison to the relevant state-of-the-art experimental techniques.

Starting with the scheme of Ref. [1], if the source particle 1 is a spin-1/2 particle in the (unknown) state $|\psi\rangle \equiv\left|\psi^{(1)}\right\rangle=a|\uparrow\rangle+b|\downarrow\rangle$ and the entangled pair $(2,3)$ also consists of spin-1/2 particles in the singlet state $\left|\psi^{(2,3)}\right\rangle=|S\rangle \equiv \frac{1}{\sqrt{2}}(|\uparrow \downarrow\rangle-|\downarrow \uparrow\rangle)$, then the joint state of these three particles is given by the direct product $\left|\psi^{(1,2,3)}\right\rangle=$ $\left|\psi^{(1)}\right\rangle \otimes\left|\psi^{(2,3)}\right\rangle$. Rewriting $\left|\psi^{(1,2,3)}\right\rangle$ in terms of the Bell basis states $\left|b_{1}\right\rangle \equiv \frac{1}{\sqrt{2}}(|\uparrow \downarrow\rangle-|\downarrow \uparrow\rangle),\left|b_{2}\right\rangle \equiv \frac{1}{\sqrt{2}}(|\uparrow \downarrow\rangle+|\downarrow \uparrow\rangle)$, 
$\left|b_{3}\right\rangle \equiv \frac{1}{\sqrt{2}}(|\uparrow \uparrow\rangle-|\downarrow \downarrow\rangle),\left|b_{4}\right\rangle \equiv \frac{1}{\sqrt{2}}(|\uparrow \uparrow\rangle+|\downarrow\rangle)$ for particles 1 and $\quad 2$ yields $\left|\psi^{(1,2,3)}\right\rangle=\frac{1}{2}\left[\left|b_{1}^{(1,2)}\right\rangle\left(-a\left|\uparrow_{3}\right\rangle-b\left|\downarrow_{3}\right\rangle\right)+\right.$ $\left|b_{2}^{(1,2)}\right\rangle\left(-a\left|\uparrow_{3}\right\rangle+b\left|\downarrow_{3}\right\rangle\right)+\left|b_{3}^{(1,2)}\right\rangle\left(b\left|\uparrow_{3}\right\rangle+a\left|\downarrow_{3}\right\rangle\right)+\left|b_{4}^{(1,2)}\right\rangle \times$ $\left.\left.\left.\left(-b\left|\uparrow_{3}\right\rangle+a \mid\right\rfloor_{3}\right\rangle\right)\right]$. If Alice measures particles 1 and 2 in the Bell basis, Bob can reconstruct the original state $|\psi\rangle$ by applying an appropriate unitary transformation to his particle 3.

Probabilistic teleportation.-Figure 1 shows an implementation of this protocol for probabilistic teleportation of electron spins in a nanostructure consisting of two quantum dots. The electron spins are prepared in their respective ground states $\left|\psi^{(1)}\right\rangle=|\uparrow\rangle$ and $\left|\psi^{(2,3)}\right\rangle=|S\rangle$ [16] [Fig. 1(c)]. In order to convincingly demonstrate successful teleportation, one should be able to initialize electron 1 in any arbitrary superposition of spin states (not only the ground state $|\uparrow\rangle$ ). This can be achieved by coherently rotating the spin of electron 1 using electron-spin resonance (ESR) [Fig. 1(d)], which results in $\left|\psi^{(1)}\right\rangle=$ $a|\uparrow\rangle+b|\downarrow\rangle$. In the measurement depicted in Fig. 1(f), Alice can only distinguish the singlet from the triplets. If her measurement outcome is a singlet, which occurs with probability $1 / 4$, the teleportation process can be completed successfully. In order to verify that teleportation has indeed been successful, Bob can apply the inverse coherent rotation of the one used for initialization in Fig. 1(d) to his spin, which should then be measured with certainty in the $|\uparrow\rangle$ state [Fig. 1(h)]. Note that this scheme is a direct electronic analogue of the first demonstration of teleportation with polarization-entangled photons by Bouwmeester et al. [2].

Deterministic teleportation. - In order to achieve deterministic quantum teleportation, Alice has to perform a full

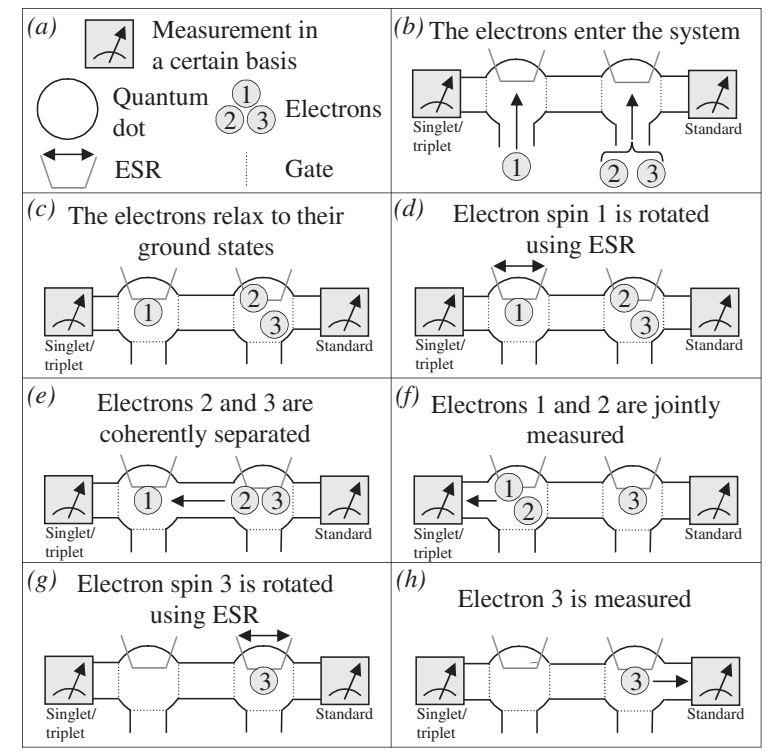

FIG. 1. Schematic setup for probabilistic quantum teleportation of an electron spin. (a): Explanation of symbols used, $\mathrm{ESR}=$ electron-spin resonance. (b) $-(\mathrm{d})$ : Initialization. (e)(f): Joint measurement of spins 1 and 2 in the singlet-triplet basis. (g): Reconstruction. (h): Readout.
Bell measurement on her two particles. For electron spins in quantum dots, however, no measurement technique is available for full Bell measurements: Current techniques allow one to distinguish singlet from triplets and to perform full measurements in the standard basis $[17,18]$. We therefore use an idea proposed by Brassard et al. [19], which consists of first transforming from the Bell basis to the standard basis and then measuring the spins in the latter basis [20]. The main part of the following analysis is devoted to finding the most efficient implementation of this procedure. We first note that the original protocol in Ref. [1] is a special case of a more general teleportation protocol. This general protocol allows Alice to freely choose her measurement basis, as long as it is a maximally entangled basis. The reason behind this freedom is that any maximally entangled basis can be used to measure the particles 1 and 2 without gaining any information on the state of the source particle. This freedom can be exploited to obtain a more efficient sequence of operations for Alice's measurement. We now first describe the general form of a two-qubit operator $U \in \mathrm{SU}(4)$ which transforms the standard basis into a maximally entangled basis (in our teleportation scheme, we need $U^{\dagger}$ ). We then derive the most efficient translation of this general form into singlequbit rotations and two-qubit $(\sqrt{\text { SWAP }})$ operations [21].

Any operator $U \in \mathrm{SU}(4)$ which transforms the standard basis into a maximally entangled basis has one of the forms $U_{1}, U_{2}$, or $U_{3}$, where

$$
\begin{aligned}
U_{1} \equiv & K_{1} e^{(1 / 4) i \pi \sigma_{x} \otimes \sigma_{x}} R_{x}^{(1)}(\mu) R_{x}^{(2)}(\nu) R_{z}^{(1)}(\xi) R_{z}^{(2)}(\zeta), \\
U_{2} \equiv & K_{1} e^{(1 / 4) i \pi\left(\sigma_{x} \otimes \sigma_{x}+\epsilon \sigma_{z} \otimes \sigma_{z}\right)} \\
& \times R_{z}^{(1)}(\xi) R_{z}^{(2)}(\zeta), \quad \text { with } \epsilon \in(0,1), \\
U_{3} \equiv & K_{1} e^{(1 / 4) i \pi\left(\sigma_{x} \otimes \sigma_{x}+\sigma_{z} \otimes \sigma_{z}\right)} R_{y}^{(1)}(\mu) R_{y}^{(2)}(\nu) \\
& \times R_{z}^{(1)}(\xi) R_{z}^{(2)}(\zeta),
\end{aligned}
$$

with $K_{1} \in \mathrm{SU}(2) \otimes \mathrm{SU}(2), \sigma_{i}(i=x, y, z)$ the Pauli matrices, $R_{n}^{(1)}(\mu)$ a rotation of qubit 1 by an angle $\mu$ around axis $n$, and $\mu, \nu, \xi, \zeta, \in[0,2 \pi)$. The mathematical proof of Eq. (1) makes use of the theory of Lie groups and Lie algebras and relies on the so-called $K_{1} A K_{2}$ decomposition theorem, which says that every $U \in \mathrm{SU}(4)$ can be decomposed as $K_{1} e^{i \alpha \sigma_{x} \otimes \sigma_{x}+i \beta \sigma_{y} \otimes \sigma_{y}+i \gamma \sigma_{z} \otimes \sigma_{z}} K_{2}$, with $K_{1}, K_{2} \in$ $\mathrm{SU}(2) \otimes \mathrm{SU}(2)$ and $\alpha, \beta, \gamma \in[0,2 \pi)$ [22]. The full proof of Eq. (1) is given in Ref. [23]. Here we proceed to translate the operators in Eq. (1) into the shortest possible sequence of single-spin rotations and $\sqrt{\text { SWAP }}$ operations. Starting with the former, $R_{n}^{(1)}(\alpha)$ and $R_{n}^{(2)}(\alpha)$ are given by

$$
R_{n}^{(1)}(\alpha)=e^{-(1 / 2) i \alpha \vec{n} \cdot \vec{\sigma} \otimes I}, \quad R_{n}^{(2)}(\alpha)=e^{-(1 / 2) i \alpha I \otimes \vec{n} \cdot \vec{\sigma}},
$$

with $\vec{n} \equiv(\sin \theta \cos \phi, \sin \theta \sin \phi, \cos \theta)$ a unit vector on the Bloch sphere $[\theta \in[0, \pi), \phi \in[0,2 \pi)], \vec{\sigma} \equiv\left(\sigma_{x}, \sigma_{y}, \sigma_{z}\right)$, and $I$ the identity matrix. Equation (2) originates from the evolution operator $U_{R}(t)=\exp \left[-(i / \hbar) \int_{0}^{t} H_{R}(\tau) d \tau\right]$ and 
corresponds to an ESR pulse applied to a single spin which is described by the Hamiltonian $\mathcal{H}_{R}(t)=-\frac{1}{2} \hbar \gamma \vec{B}(t) \cdot \vec{\sigma}$ [ $\gamma$ is the gyromagnetic ratio and $\alpha \equiv-\gamma \int_{0}^{t} B(\tau) d \tau$ ]. Similarly, the $\sqrt{\text { SWAP }}$ operation for electron spins in quantum dots originates from the exchange interaction described by the Heisenberg Hamiltonian $\mathcal{H}_{\mathrm{EX}}(t)=$ $(1 / 4) \hbar^{2} J(t) \vec{\sigma}^{(1)} \cdot \vec{\sigma}^{(2)}$, with $J(t)$ the time-dependent exchange energy, the energy difference between singlet and triplets. The time evolution operator $U_{\mathrm{EX}}(t)$ corresponding to $\mathcal{H}_{\mathrm{EX}}(t)$ or, equivalently, $\mathrm{EX}(\beta) \equiv U_{\mathrm{EX}}(t)$, is given by

$\operatorname{EX}(\beta)=e^{-(1 / 4) i \beta}\left(\begin{array}{cccc}e^{(1 / 2) i \beta} & 0 & 0 & 0 \\ 0 & \cos \left(\frac{1}{2} \beta\right) & i \sin \left(\frac{1}{2} \beta\right) & 0 \\ 0 & i \sin \left(\frac{1}{2} \beta\right) & \cos \left(\frac{1}{2} \beta\right) & 0 \\ 0 & 0 & 0 & e^{(1 / 2) i \beta}\end{array}\right)$.

Here $\beta(t) \equiv-\hbar \int_{0}^{t} J(\tau) d \tau$. $\operatorname{EX}(\pi)$ represents the SWAP operation which exchanges the states of two spins. An interaction switched on for half this time, $\operatorname{EX}(\pi / 2)$, is called the $\sqrt{\text { SWAP }}$ operation.

Regarding $U_{1}-U_{3}$ in Eq. (1), we first note that $\exp \left[(1 / 4) i \pi \sigma_{x} \otimes \sigma_{x}\right]$ cannot be expressed as a single $\sqrt{\text { SWAP }}$ operation plus rotations. This can directly be seen from the form of the matrix $(\sqrt{\mathrm{SWAP}})^{\dagger} \exp \left[(1 / 4) i \pi \sigma_{x} \otimes\right.$ $\left.\sigma_{x}\right] . U_{1}-U_{3}$ can, however, be expressed in terms of two $\sqrt{\text { SWAP }}$ operations as

$$
U \equiv \sqrt{\mathrm{SWAP}} R_{n_{\phi}}^{(1)}(\pi) \sqrt{\mathrm{SWAP}},
$$

with $\vec{n}_{\phi} \equiv(\cos \phi, \sin \phi, 0)$ [24]. Equation (3) is the optimal decomposition of Eq. (1) in terms of the number of required operations [25]. Rewriting $U$ as $U^{\prime} \equiv$ $-\exp [-(1 / 4) i \pi] U$, we find that $U^{\prime}$ transforms the standard basis into the maximally entangled basis

$$
\begin{aligned}
U^{\prime}|\uparrow \uparrow\rangle & =\frac{1}{\sqrt{2}}(i|\uparrow \downarrow\rangle+|\downarrow \uparrow\rangle), \\
U^{\prime}|\uparrow \downarrow\rangle & =\frac{1}{\sqrt{2}}\left(i e^{-i \phi}|\uparrow \uparrow\rangle+e^{i \phi}|\downarrow \downarrow\rangle\right), \\
U^{\prime}|\downarrow \uparrow\rangle & =\frac{1}{\sqrt{2}}\left(e^{-i \phi}|\uparrow \uparrow\rangle+i e^{i \phi}|\downarrow \downarrow\rangle\right), \\
U^{\prime}|\downarrow \downarrow\rangle & =\frac{1}{\sqrt{2}}(|\uparrow \downarrow\rangle+i|\downarrow \uparrow\rangle) .
\end{aligned}
$$

After Alice has applied $U^{\prime \dagger}$ to her two qubits, the appro- priate unitary transformations which Bob has to perform in order to reconstruct the original quantum state can be calculated using the approach in Ref. [26] and are given by ( $Q_{1}$ corresponds to measurement outcome $|\uparrow \uparrow\rangle, Q_{2}$ to $|\uparrow \downarrow\rangle$, etc.)

$$
\begin{aligned}
& Q_{1}=\left(\begin{array}{cc}
-i & 0 \\
0 & 1
\end{array}\right), \quad Q_{2}=\left(\begin{array}{cc}
0 & i e^{-i \phi} \\
-e^{i \phi} & 0
\end{array}\right), \\
& Q_{3}=\left(\begin{array}{cc}
0 & e^{-i \phi} \\
-i e^{i \phi} & 0
\end{array}\right), \quad Q_{4}=\left(\begin{array}{cc}
-1 & 0 \\
0 & i
\end{array}\right) .
\end{aligned}
$$

Translating $Q_{i}$ into single-spin rotations in the $(x, y)$ plane [27], we obtain $Q_{1}=R_{n_{\phi}}(\pi / 2) R_{n_{\phi}^{\perp}}(\pi / 2) R_{n_{\phi}}(-\pi / 2)$ and similar results for $Q_{2}-Q_{4}$; see Table I. By applying these transformations, the original quantum state is reconstructed and remains available for further processing. In order to demonstrate that the teleportation process has been successful, one could perform an analogous experiment as depicted in Fig. 1(g), where Bob rotates the spin of his particle after receiving Alice's message such that it ends up as $|\uparrow\rangle$ or $|\downarrow\rangle$. He should then measure with certainty $|\uparrow\rangle$ (respectively, $|\downarrow\rangle$ ). The appropriate rotations Bob needs to apply in this case are also given in Table I. The quantum state of the source particle is destroyed in this experiment, so it cannot be used for further processing. Figure 2 shows how the setup of Fig. 1 has to be modified for deterministic teleportation. All steps of the protocol are the same as in Fig. 1, except for Alice's measurement in Fig. 1(f), which now consists of the operation $U$ [Eq. (3)] followed by a readout of the two spins in the standard basis. Note that this scheme is, in principle, also suitable for entanglement swapping [28] if one starts out with two pairs of entangled spins in separate dots: By bringing together and entangling one spin of each pair and performing a Bell measurement on this newly formed pair, the remaining (separated) spins, which have never met, become entangled.

Feasibility.-In this last section, we discuss the feasibility of the proposed teleportation schemes. We first note that all of the ingredients (double quantum dots occupied by two entangled electron spins [13], ESR rotations [29], $\sqrt{\text { SWAP }}$ operations [14], single-shot readout techniques for

\begin{tabular}{|c|c|c|c|}
\hline Alice's result & Reconstruction of original state & Measure $|\uparrow\rangle$ & Measure $|\downarrow\rangle$ \\
\hline$|\uparrow \uparrow\rangle$ & $R_{n_{\phi}}\left(\frac{\pi}{2}\right) R_{n_{\phi}^{\perp}}\left(\frac{\pi}{2}\right) R_{n_{\phi}}\left(-\frac{\pi}{2}\right)$ & $R_{n_{\phi}^{\perp}}(\rho)$ & $R_{n_{\phi}^{\perp}}(\rho+\pi)$ \\
\hline$|\uparrow \downarrow\rangle$ & $R_{n_{\phi}}\left(\frac{\pi}{2}\right) R_{n_{\phi}^{\perp}}\left(\frac{\pi}{2}\right) R_{n_{\phi}}\left(\frac{\pi}{2}\right)$ & $R_{n_{\phi}^{\perp}}(-\rho+\pi)$ & $R_{n_{\phi}^{\perp}}(-\rho)$ \\
\hline$|\downarrow \uparrow\rangle$ & $R_{n_{\phi}}\left(\frac{\pi}{2}\right) R_{n_{\phi}^{\perp}}\left(-\frac{\pi}{2}\right) R_{n_{\phi}}\left(\frac{\pi}{2}\right)$ & $R_{n_{\phi}^{\perp}}(\rho+\pi)$ & $R_{n_{\phi}^{\perp}}(\rho)$ \\
\hline$|\downarrow \downarrow\rangle$ & $R_{n_{\phi}}\left(-\frac{\pi}{2}\right) R_{n_{\phi}^{\perp}}\left(\frac{\pi}{2}\right) R_{n_{\phi}}\left(\frac{\pi}{2}\right)$ & $R_{n_{\phi}^{\perp}}(-\rho)$ & $R_{n_{\phi}^{\perp}}(-\rho+\pi)$ \\
\hline
\end{tabular}
electron spins $[17,18,30])$ are already available. Comparing the duration of an ESR rotation $\sim 50 \mathrm{~ns}$ for magnetic fields of $1 \mathrm{mT}$ [31] to the duration of a $\sqrt{\mathrm{SWAP}}$

TABLE I. Spin rotations in the $(x, y)$ plane which Bob has to apply as a function of Alice's measurement outcome (first column) in order to reconstruct the original quantum state (second column) and measure with certainty $|\uparrow\rangle$ (third column) or $|\downarrow\rangle$ (fourth column) if particle 1 has been rotated by an angle $\rho$ [Fig. (1(d)]. $n_{\phi}^{\perp}$ denotes the axis in the $(x, y)$ plane perpendicular to $n_{\phi}$. 


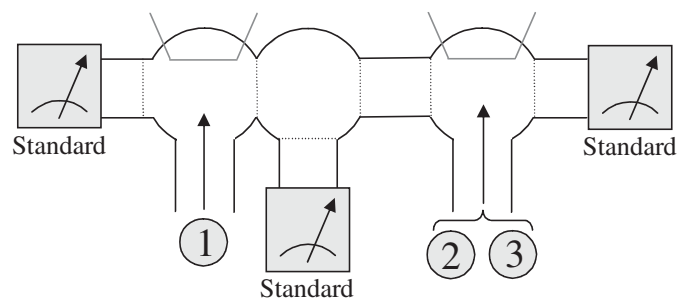

FIG. 2. Quantum dot setup for deterministic teleportation of an electron. See the text for explanation.

operation $\sim 180$ ps [14] and assuming a fast readout, we see that the total time duration of the teleportation process is determined mainly by the ESR rotations. For the probabilistic scheme, we then find $t_{\text {prob }} \sim 100 \mathrm{~ns}$ (corresponding to two rotations) and for the deterministic scheme $t_{\mathrm{det}} \sim$ 150-250 ns (depending on whether Bob measures the spin of his particle or not). Teleportation can thus be completed within the single-spin relaxation time $T_{1} \sim 1 \mathrm{~ms}$ [17]. Whether it can also be completed before decoherence sets in is as yet unknown. Recent measurements indicate that $T_{2}$ is limited by hyperfine interaction in a GaAs quantum dot and report times $T_{2}^{*} \sim 10 \mathrm{~ns}$ [14,32]. However, using quantum control techniques such as spin echo, these times can be extended to $>1 \mu$ s [14]. This technique has also been employed in one of the teleportation experiments with ions [5].

In conclusion, we have proposed a scheme for deterministic teleportation of electrons in a quantum dot nanostructure which involves the shortest possible sequence of single-spin rotations and two-qubit $(\sqrt{\mathrm{SWAP}})$ operations. Because of the high level of control over the system, teleportation can, in principle, be achieved with a $100 \%$ success rate at the "push of a button," as in the experiments with ions [33]. To actually perform the proposed experiment is challenging, mainly due to the required fast coherent rotations and readout, but does not seem unfeasible.

Stimulating discussions with L.P. Kouwenhoven, L. M. K. Vandersypen, and I. T. Vink are gratefully acknowledged. This work has been supported by the Netherlands Organisation for Scientific Research (NWO) and by the EU's Human Potential Research Network under Contract No. HPRN-CT-2002-00309 ("QUACS").

[1] C. H. Bennett et al., Phys. Rev. Lett. 70, 1895 (1993).

[2] D. Bouwmeester et al., Nature (London) 390, 575 (1997); D. Boschi et al., Phys. Rev. Lett. 80, 1121 (1998); J.-W. Pan et al., ibid. 86, 4435 (2001); E. Lombardi et al., Phys. Rev. Lett. 88, 070402 (2002); I. Marcikic et al., Nature (London) 421, 509 (2003); D. Fattal et al., Phys. Rev. Lett. 92, 037904 (2004); Z. Zhao et al., Nature (London) 430, 54 (2004); R. Ursin et al., ibid. 430, 849 (2004).

[3] M. A. Nielsen, E. Knill, and R. Laflamme, Nature (London) 396, 52 (1998).

[4] A. Furusawa et al., Science 282, 706 (1998).
[5] M. Riebe et al., Nature (London) 429, 734 (2004); M. D. Barrett et al., ibid. 429, 737 (2004).

[6] J.H. Reina and N.F. Johnson, Phys. Rev. A 63, 012303 (2001).

[7] Y. N. Chen et al., New J. Phys. 7, 172 (2005).

[8] O. Sauret, D. Feinberg, and T. Martin, Eur. Phys. J. B 32, 545 (2003).

[9] C. W. J. Beenakker and M. Kindermann, Phys. Rev. Lett. 92, 056801 (2004).

[10] F. de Pasquale, G. Giorgi, and S. Paganelli, Phys. Rev. Lett. 93, 120502 (2004).

[11] M. N. Leuenberger, M.E. Flatté, and D. D. Awschalom, Phys. Rev. Lett. 94, 107401 (2005).

[12] But the scheme can also be applied to other systems with three qubits.

[13] M. Ciorga et al., Phys. Rev. B 61, R16315 (2000); J. M. Elzerman et al., ibid. 67, 161308(R) (2003).

[14] J. R. Petta et al., Science 309, 2180 (2005).

[15] For a recent review on few-electron quantum dots as spin qubits, see J. M. Elzerman et al., Lect. Notes Phys. 667, 25 (2005).

[16] R. Hanson et al., Phys. Rev. B 70, 241304(R) (2004).

[17] J. M. Elzerman et al., Nature (London) 430, 431 (2004).

[18] R. Hanson et al., Phys. Rev. Lett. 94, 196802 (2005).

[19] G. Brassard, S.L. Braunstein, and R. Cleve, Physica (Amsterdam) 120D, 43 (1998).

[20] This approach was also used in the NMR and ion experiments in Refs. [3,5].

[21] These are universal operations for quantum computation with electron spins in quantum dots; see G. Burkard, D. Loss, and D. P. DiVincenzo, Phys. Rev. B 59, 2070 (1999).

[22] B. Kraus and J. I. Cirac, Phys. Rev. A 63, 062309 (2001); N. Khaneja and S. J. Glaser, Chem. Phys. 267, 11 (2001).

[23] R. L. de Visser et al. (to be published).

[24] Equation (3) corresponds to, for example, Eq. (1a) by setting $\phi=0, K_{1}=\exp (-i \pi / 4) I, \mu=\pi$, and $\nu=\xi=$ $\zeta=0$.

[25] See also J. Zhang et al., Phys. Rev. Lett. 91, 027903 (2003), in which an upper bound is derived for the number of two-qubit operations that are required for implementing quantum gates of the form $\exp \left[i \gamma \sigma_{z} \otimes \sigma_{z}\right]$, with $\gamma \in$ $(0, \pi / 4]$.

[26] C. Li, H.-S. Song, and Y.-X. Luo, Phys. Lett. A 297, 121 (2002).

[27] Here we assume the Zeeman-splitting field to be in the $z$ direction, so that spin rotations are performed in the $(x, y)$ plane.

[28] B. Yurke and D. Stoler, Phys. Rev. Lett. 68, 1251 (1992); M. Zukowski et al., ibid. 71, 4287 (1993).

[29] F. H. L. Koppens et al. (unpublished).

[30] In the probabilistic scheme, Alice's measurement can be done straightforwardly by tuning the energy levels in the left dot in Fig. 1(e) such that only the singlet level is available for the incoming electron 2.

[31] L. M. K. Vandersypen et al., in Quantum Computing and Quantum Bits in Mesoscopic Systems, edited by A. Leggett, B. Ruggiero, and P. Silvestrini (Springer, New York, 2004).

[32] F. H. L. Koppens et al., Science 309, 1346 (2005).

[33] H. J. Kimble and S. J. van Enk, Nature (London) 429, 712 (2004). 